\title{
Gene cloning and gene expression characteristics of alcohol dehydrogenase in Osmanthus Fragrans var. Semperflorens
}

\author{
Lili Chen', Zihan Wang', Rong Chen ${ }^{1}$, Hanjie He', Jinghua $\mathrm{Ma}^{2}$, Dangquan Zhang ${ }^{2 *}$ \\ ${ }^{1}$ College of Life Science and Technology, Central South University of Forestry and Technology, Changsha 410004, China, ${ }^{2}$ College of Forestry, \\ Henan Agricultural University, Zhengzhou 450002, China
}

\section{A B S T R A C T}

\begin{abstract}
Because of a lot of aroma components in the flowers, Osmanthus fragrans is widely used as ornamental tree species and its flowers is used as materials of spices and food additives. Alcohol dehydrogenase (ADH) plays an important regulation role in growth, development, stress resistance and aroma synthesis of plants. Using the flowers of $O$ fragrans var. semperflorens as materials, the full-length cDNA of ADH gene (termed OfADH) was successfully cloned through rapid amplification of cDNA ends (RACE), which has the 1128 bp open reading frame (ORF) in length and codes 375 amino acids. The results of conserved domain analysis show that the $O f A D H$ belongs to the zinc-dependent medium-chain dehydrogenase/reductase (MDR) superfamily, contains 22 NAD(P) binding sites in the liver_alcohol_DH_like conserved domains, and also has the PLN02740 (Alcohol dehydrogenase-like) domain. The results of semi-quantitative RT-PCR indicate that the expression level of $O f A D H$ in $O$. fragrans flowers is consistent with the rule of aroma formation and release, while the expression of $O f A D H$ in leaves is positively correlated with high temperature stress, suggesting that $O f A D H$ has obvious functional diversity in regulation of aroma synthesis and response to high temperature stress.
\end{abstract}

Keywords: Osmanthus fragrans var. semperflorens; Alcohol Dehydrogenase; Aroma synthesis

\section{INTRODUCTION}

Alcohol dehydrogenase (ADH), a zinc metalloprotease, existing in all kinds of organs and cells of plants, animal and microorganisms widely (Jacquelyn LM et al., 2013; Kluver N et al., 2014), is one of the rates limiting enzyme of short chain alcohols metabolism in organism (Cheung et al., 2017; Naser and Lawrey, 2018). In the presence of NAD+ and NADH, $\mathrm{ADH}$ is able to catalyze a reversible reaction between primary alcohols and aldehydes, also between ethanol and aldehydes (Gottlieb et al., 1982). ADH plays an important role in the growth and development process of humans, animals and plants. It exhibits obvious biological functional diversity (Alpat et al., 2010). So there are many researchers have carried out researches on the functionality application and gene regulation mechanism of ethanol dehydrogenase (DiMeglio, et al., 2014), including the ethanol biosensor (Niculescu et al., 2002), drug therapy (Vanessa, et al., 2014), disease diagnosis (Charlton et al., 2002), fruit storage (Ning et al., 2009), fruit flavor improved (Ting et al., 2012), and stress and resistance in plants (Liang et al., 2012; Liu et al., 2007).
In recent years, studies have indicated that alcohol dehydrogenase played an important role in the formation and diversity of plant aroma (Kmita et al., 2018; Liu and Liu, 2010; Shen et al., 2017). The main ingredients of plant fragrance are terpenoids (isoprene, monoterpene and sesquiterpene), fatty acid derivatives (volatile aldehydes and alcohols) and phenyl/phenylpropanoid substances, that are synthesized respectively by the terpenoid metabolism pathway, the fatty acid derivatives metabolic pathway and the phenylpropanoid pathway (Zhang Qiang, 2009). In the fatty acid derivatives metabolic pathway of apple aroma (Robert, et al., 2007), hexanal transformed by linoleic acid hydroperoxide could generate 2-methylbutanol which can produce 2-isoamyl acetate by the alcohol acyltransferase in presence of ADH (Dudareva et al., 2008). And also, in the mature process of fruits, the expression level of $\mathrm{ADH}$ has important influence on the diversity of aroma components, especially the content of aldehydes and alcohols (Iyit, 2018; Jiang et al., 2018; Khan et al., 2018). Studies have indicated that the increase of ethylene content would lead to lower expression of ADH (Defilippi et al., 2005), while 
the increase of alcohols would make genetically modified fruit more flavor (Speirs J et al., 1998).

Osmanthus fragrans Lour is a traditional flower in China. Because of its unique scent, commercial extracts are in high demand for use in the production perfumes and cosmetics, such as flavouring tea, wine, and foods. O. fragrans var. semperflorens, that is a special variety of $O$. Fragrans with more longer flowering period than others, could be flowering in all four seasons (Khan, 2018; Sultana et al., 2018; Kibria et al., 2018). The flowers of $O$. fragrans var. semperflorens are collected as materials for $O f A D H$ full-length cDNA cloning and expression analysis. This study will provide the basis for exploring the regulation mechanism and biological mechanism of ADH gene in aroma synthesis of $O$. fragrans var. semperflorens.

\section{MATERIALS AND METHODS}

\section{Materials}

The tissues of O. fragrans var. semperflorens (Fig.1) were collected from the plant garden of Central South University of Forestry and Technology in 2014 and 2015.

Flowers and leaves are collected separately in March, June, September and December. The flowers in different flowering stages and the blooming flowers in 18:00, 22:00, next 2:00, $6: 00,10: 00,14: 00$ also have been collected. All of materials have been stored in $-80^{\circ} \mathrm{C}$ low temperature refrigerator.

\section{Methods}

RNA extraction and First-strand cDNA synthesis

Total RNA was extracted by modified CTAB combined with plant RNA Kit (Omega) and detected by 1\% agarose gel electrophoresis. First-strand cDNA synthesis was performed using the Invitrogen SuperScript ${ }^{\mathrm{TM}}$ III Reverse Transcriptase.

\section{O.fragrans $A D H$ gene cloning}

The main fragment of $O f A D H$ was amplified with the primers (Table. 1). The PCR product was purified and inserted into pMD-18 $\mathrm{T}$ vector (TaKaRa) and sequenced. To clone the full-length $O f A D H$, the 5'-end and 3'-end were obtained by RACE-PCR (Invitrogen 3' RACE/5' RACE System for Rapid Amplification of cDNA Ends). The full-length cDNA of $O f A D H$ was finally amplified using the primers Fl (Table. 1). The PCR products were cloned into $\mathrm{pMD}-18 \mathrm{~T}$ vector for squencing.

\section{Bioinformatics analysis}

The full-length $O f A D H$ sequence was analyzed by BioEdit. The DNAMAN soft had been used to carry out homology sequence alignment between $O$. fragrans and other plants. All of plant ADH genes were searched in the NCBI Genbank. Phylogenetic tree was built by MEGA4 in NJ component, and tested 1000 times by bootstrap analysis. The protein secondary structure was analyzed online (http://web. expasy.org/protscale/). The protein conserved domain of ADH was also analyzed and forecasted by online software (http://www.ncbi.nlm.nih.gov/Structure/cdd/wrpsb.cgi). Searching similar sequence in PBD (http://www.rcsb. org/pdb/home.do) according to the OfADH amino acid sequence. Through homology modeling by Swiss-model (http://swissmodel.expasy.org/) in alignment mode, the three-level structure of OfADH protein was predicted.

Gene expression pattern by Semi-quantitative RT-PCR $O f A D H$ mRNA expression in different seasons, different flowering stages and different times a day were evaluated by semi-quantitative RT-PCR analysis. The $O f A D H$ mRNA transcript was amplified with specific primers (BDADH-F:5 '-CTCTGTTTCCTCGGATTCCC-3 BDADH-R:5'; '-CCCCCAGTCCACCTGTAGTC-3') designed from the conserved region of OfADH cDNA. A parallel amplification with specific primers (18s-F: 5'-TCTAAATCCCTTAACGAGGATC-3'; 18s-R: 5'-CTATGAAATACGAATGCCCC-3') for 18s gene employed as an internal control to show that equal amount of cDNA was used in each PCR (Susanne B. et al, 2010). The Quantity One had been used to carry out the gray level analysis for semi-quantitative RT-PCR results.

\section{RESULTS AND ANALYSIS}

\section{Cloning and sequence analyses of $\mathrm{OFADH}$}

The main fragment of $O f A D H$ was $828 \mathrm{bp}, 3$ '-end fragments was 475bp, and 5'-end fragrans was 393bp. The full-length of $O f A D H$ containing a whole open reading frame was 1295bp (Fig. 2).
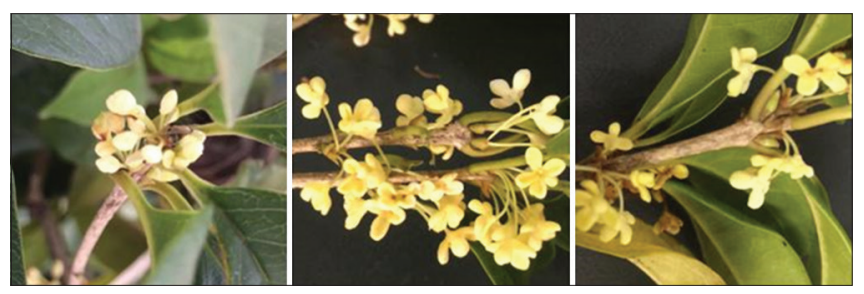

Fig 1. Flowering of Osmanthus fragrans Var. Semperflorens. 1. Flower buds; 2 . Full flowering; 3. Flowering drop-off.

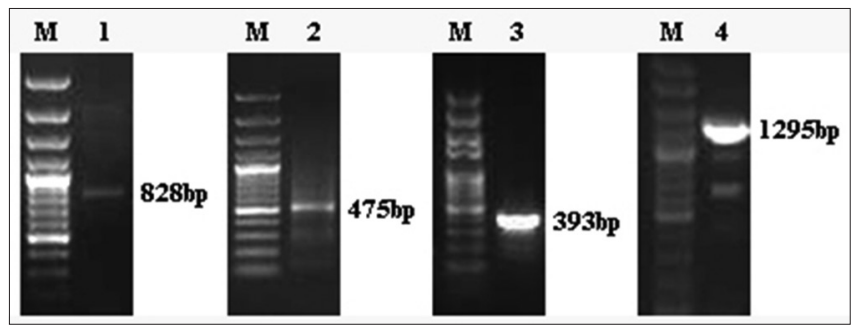

Fig 2. Full-length cDNA cloning of OfADH. M: DNA Marker; 1: Amplification product of conserved region; 2: Amplification product of 3' RACE; 3: Amplification product of 5' RACE; 4: Amplification production of DXPS ORF. 


\begin{tabular}{|c|c|}
\hline 1 & CAGCCCACCCCGCACGGG ACAATAT TCTCCCAT TTGCAGCCCAG IATCCACC ATGACI AGCGGCAATCCAGGAGTAAT TACA TGCAAAGC \\
\hline 1 & 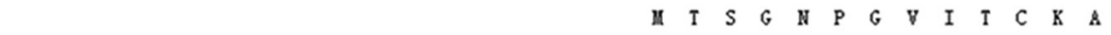 \\
\hline 91 & TGCGG TGG TATGG AARTCAGGAGAGCCAT TGARAG TAG AGGARATACARG TGGATCCTCCCARATCCTCTGARG TTAGGATCARARTGCT \\
\hline 14 & 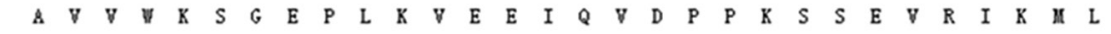 \\
\hline 181 & 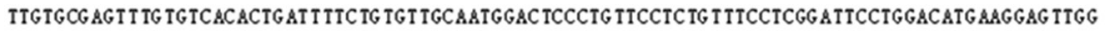 \\
\hline 84 & $\begin{array}{cccccccccccccccccccccccccccccc}C & A & S & L & C & H & \text { I } & D & F & \text { L } & C & C & N & G & \text { L } & \text { P } & \nabla & \text { P } & \text { L } & \text { F } & \text { P } & R & \text { I } & P & G & H & E & G & \nabla & G\end{array}$ \\
\hline 271 & CATGATAGAGAGTG TGGGAGAAGGAG TCACAAATCTGARAGAAGGAGATATAG TGATGCCACTCTATCTGGGAGAATGTGGGGASTGTCT \\
\hline 74 & 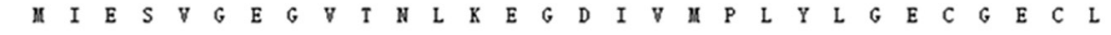 \\
\hline 361 & GAAT TGCAAA TCTGGGAAGACAAAT T TATGCCACAAATATCCCT TAAATT TCACTGGCCTAATGCCAGATGGCACATCAAGABATCACAT \\
\hline 104 & 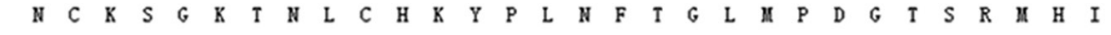 \\
\hline 451 & TGGAGATCAG ATAATRTATCACCATTITAGCTGTGGTACATGG TCTGARTATG ITG TT ATCGATGCGAATITIGCAG TCAAGGTIGATCC \\
\hline 134 & 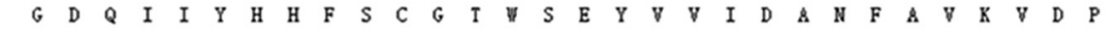 \\
\hline 541 & 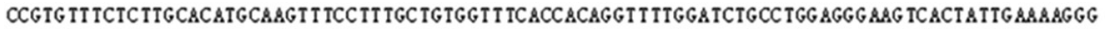 \\
\hline 164 & 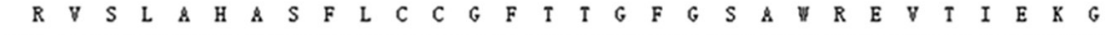 \\
\hline 631 & CTCAACTATTGCTGITATTGGTG TTGGTGCTG TTGGACTCGGAG TGRTAG AGGCAGCGCGABTGARTGGAGCT TCTAGGATARTIGGGAT \\
\hline 194 & 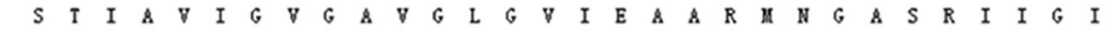 \\
\hline 721 & TGACATAAACAACTTGAAGAAGAAGAABGGAGAAGCCTITGGAATGACTGARTTTATCAATCCAABAGAATCTGATARACCTG TATCAGA \\
\hline 224 & 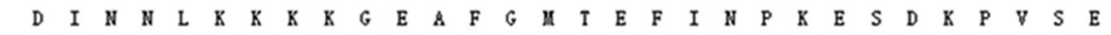 \\
\hline 811 & AT TGATTAABG ATACTACAGGTGGACTGGGGG TGGAT TACTGT TATGAG TGCACTGGAG ITCCAG BGCTGC TAAATG ABGCABTTG AGGG \\
\hline 254 & $\begin{array}{lllllllllllllllllllllllllllllll}\mathrm{L} & I & \mathrm{~K} & \mathrm{D} & \mathrm{T} & \mathrm{T} & G & G & \mathrm{~L} & G & \mathrm{~V} & \mathrm{D} & \mathrm{Y} & \mathrm{C} & \mathrm{Y} & \mathrm{E} & \mathrm{C} & \mathrm{T} & G & \mathrm{~V} & \mathrm{P} & \mathrm{E} & \mathrm{L} & \mathrm{L} & \mathrm{N} & \mathrm{E} & \mathrm{A} & \mathrm{I} & \mathrm{E} & G\end{array}$ \\
\hline 901 & ATCCARGG TGGGACTTGGGACARTAG IT T TCATTGGTGCGGGACTTCATT TRAGTGGGGAGCTCARA TACATTCOCC TCT TG TGTGGTAG \\
\hline 284 & 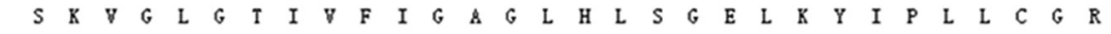 \\
\hline 991 & 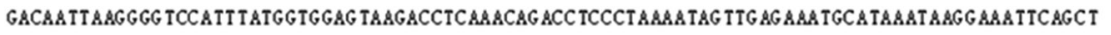 \\
\hline 314 & 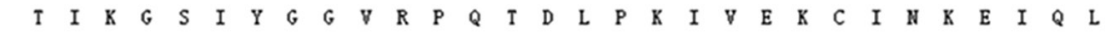 \\
\hline 108 & 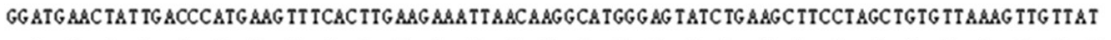 \\
\hline 344 & 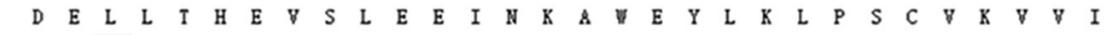 \\
\hline 1171 & 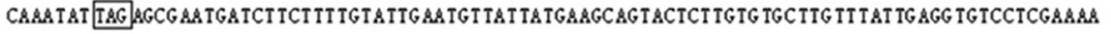 \\
\hline 374 & $\mathrm{~K}$ \\
\hline 1261 & CTAOGTI TTOCTT TGGATT TARTAAAGAATGGAGAAART TAATGAGGG \\
\hline
\end{tabular}

Fig 3. cDNA and amino acid sequence of $O$. fragrans var. semperflorens ADH.

Assembling all three fragments of $O f A D H$, we get 1308bp full-length cDNA. OfADH has an open reading frame of 1128bp (53bp-1180bp), encoding a peptide of 375 animo acids (Fig. 3).

\section{Analysis of $O f A D H$ protein structure}

$O f A D H$ protein has an estimated molecular weight of 40497.11 Da with an isoelectric point of 5.9. Its formula is $\mathrm{C}_{1806} \mathrm{H}_{2886} \mathrm{~N}_{470} \mathrm{O}_{532} \mathrm{~S}_{25}$. Analyzed in Hphob./Kyte \& Doolittle model, this protein is a hydrophobic lipid soluble protein with grand average of hydropathicity 0.092 and aliphatic index 96.88 (Fig. 4). There are 21.07\% alpha helix, $32.53 \%$ extended strand, $14.93 \%$ beta turn, and $31.47 \%$ random coil in $O f A D H$ protein structure.

As shown in Fig. 4, OfADH protein belongs to the zincdependent medium-chain dehydrogenase/reductase (MDR) superfamily, and contains $22 \mathrm{NAD}(\mathrm{P})$ binding sites, 29 dimer interface, 4 catalytic $\mathrm{Zn}$ binding site, and 4 structural $\mathrm{Zn}$ binding site in the liver_alcohol_DH_like conserved domains, and it also had the PLN02740 (Alcohol dehydrogenase-like) domain (Fig.5).

Through the template of crystal structure and dynamic structure of Arabidopsis ADH protein (4jiji.1), homo-dimer model is constructed by Swiss-Model (Fig. 6). The protein model GMQE score is 0.76 , and the similarity of template protein sequence is $45.73 \%$.

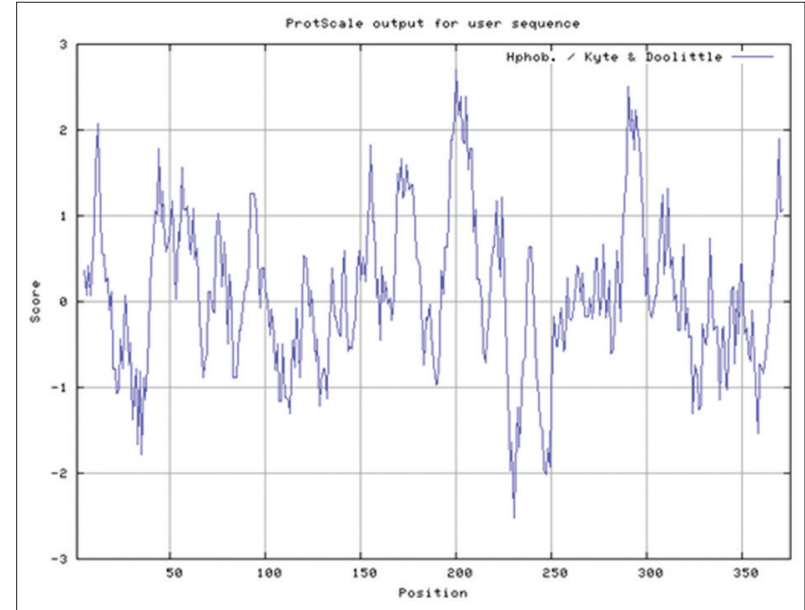

Fig 4. Profiling of hydrophobicity and hydrophilicity of $O A A D H$ protein.

\section{Multiple sequence alignment and phylogenetic tree analyses of ADH}

Multiple sequence alignment analysis reveals that $O f A D H$ protein shares $96.2 \%$ identity with the $\mathrm{ADH}$ from Oleaeuropaea (JN200815.1) (Fig. 7). Phylogenetic analysis (Fig. 8) further reveals that the $O f A D H$ protein is most closely related to ADH from Oleaeuropaea, and as a major branch of ADH1 cluster with Arabidopsis thaliana, Oryza sativa, and Zea mays. However, OfADH shares low homology of the amino acid sequence with Musa acuminata, and Populus tremuloides. According to sequence similarity and phylogenetic analyses, we presume that $\mathrm{ADH}$ family 
Table 1: Primers used for gene cloning and expression of $O$. fragrans ADH

\begin{tabular}{lll} 
Names & The primers & Nucleotide \\
\hline Cloning of the main fragment & ADH-F & TCGCAATGGACTCCCTGT \\
3'RACE & ADH-R & GGGAGGTCTGTTTGAGGTCTT \\
& ADH-3'gsp2 & CTGGAGGGAAGTCACTATTGAAAAGG \\
5' RACE & ADH-3'gsp3 & GACTGGGGGTGGATTACTGTTATGA \\
& ADH-5'gsp1 & CCACCTGTAGTATCTTTG \\
& ADH-5'gsp2 & TCCAACAGCACCAACACCAATA \\
Cloning of full-length of & ADH-5'gsp3 & ACATTCCCCACATTCTCCCA \\
OfADH & FI-ADH-F & ACGGGACAATATTCTCCCA \\
\hline
\end{tabular}

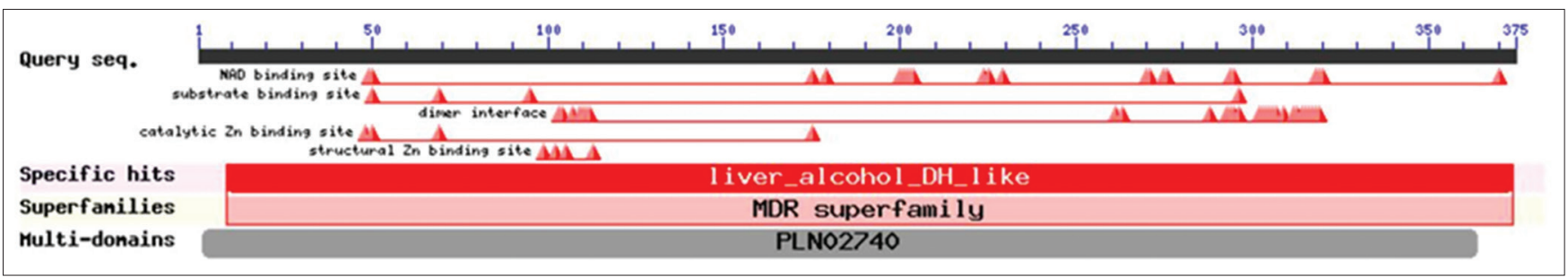

Fig 5. Profiling of conserved domains of OfADH protein.

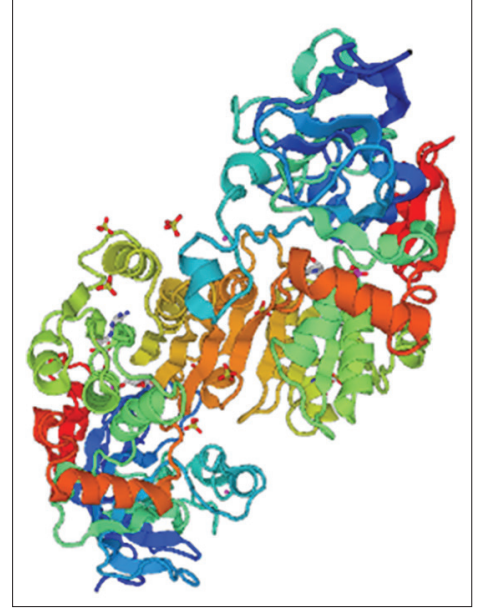

Fig 6. Prediction of three-dimensional structure of OfADH protein.

from plants represent significant differences related to their functional diversity in different plants.

\section{OfADH gene expression patterns}

The $O f A D H$ gene expression patterns of flowers and leaves in Mar, Jun, Sep and Dec were studied by semi-quantitative RT-PCR (Fig. 9). OfADH expression level of flowers was the highest in March, and then decreased significantly in June. In September, $O f A D H$ expression had rose again a bit, while the expression was similar to March in December. The $O f A D H$ expression of flowers performed a high-lowhigh pattern in different season. However, the $O f A D H$ gene expression pattern of leaves was different from flowers. OfADH expression level of leaves was the lowest in March, and then increased in June. In September, the highest expression level was reached. So, the gene expression pattern of $O f A D H$ in leaves was low-high-low trend.
During flowering, the expression of $O f A D H$ gene increased continuously from the budding stage and reached the highest expression level at the blooming stage. The expression of $O f A D H$ gene decreased rapidly from fullflowering to drop-off stage (Fig. 10).

In one of the full flowering days, the $O f A D H$ gene expression level was the highest at 14:00, which was 3 times that at 2:00 (the lowest expression level). And the expression level of the other time points was not significant (Fig. 11).

\section{DISCUSSIONS}

The studies of ADH gene about humans, animals and microorganisms were very more (Pan et al., 2014) than this about plants (Chao et al., 2014). At present, ADH gene have been identified in a lot of herbaceous and woody plants, such as Arabidopsis (Chung et al., 1999), Legumes (Fukudaet et al., 2005), Poplar (Bomatia et al., 2005), and Pyrus (Zheng et al., 2011). ADH gene are widely expressed in leaves, flowers, stems, roots and seeds, and demonstrate diversity of biological function in plants.

The interaction between $\mathrm{ADH}$ and ethylene could promote the persimmon deastringency during maturation process (Min et al., 2012). ADH gene also regulated the synthesis of fruit aroma components through the fatty acid derivatives metabolic pathway (Dudareva et al., 2008). Especially, it would affect fruit aromatic components and flavor of fruit a lot that the content of $\mathrm{ADH}$ had a significant change during fruit maturation and ripening period (Imahon et al., 2002; 


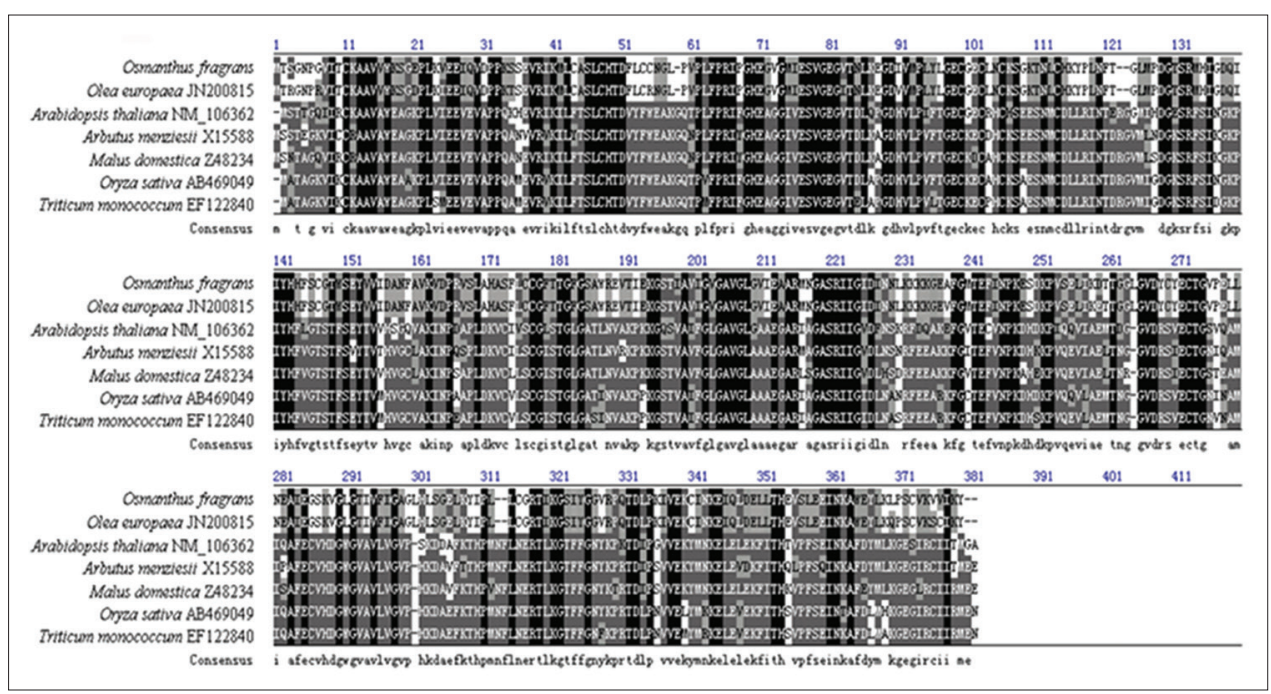

Fig 7. Amino acid multiple alignment of $\mathrm{ADH}$ s between $O$. fragrans var. semperflorens and other plants.

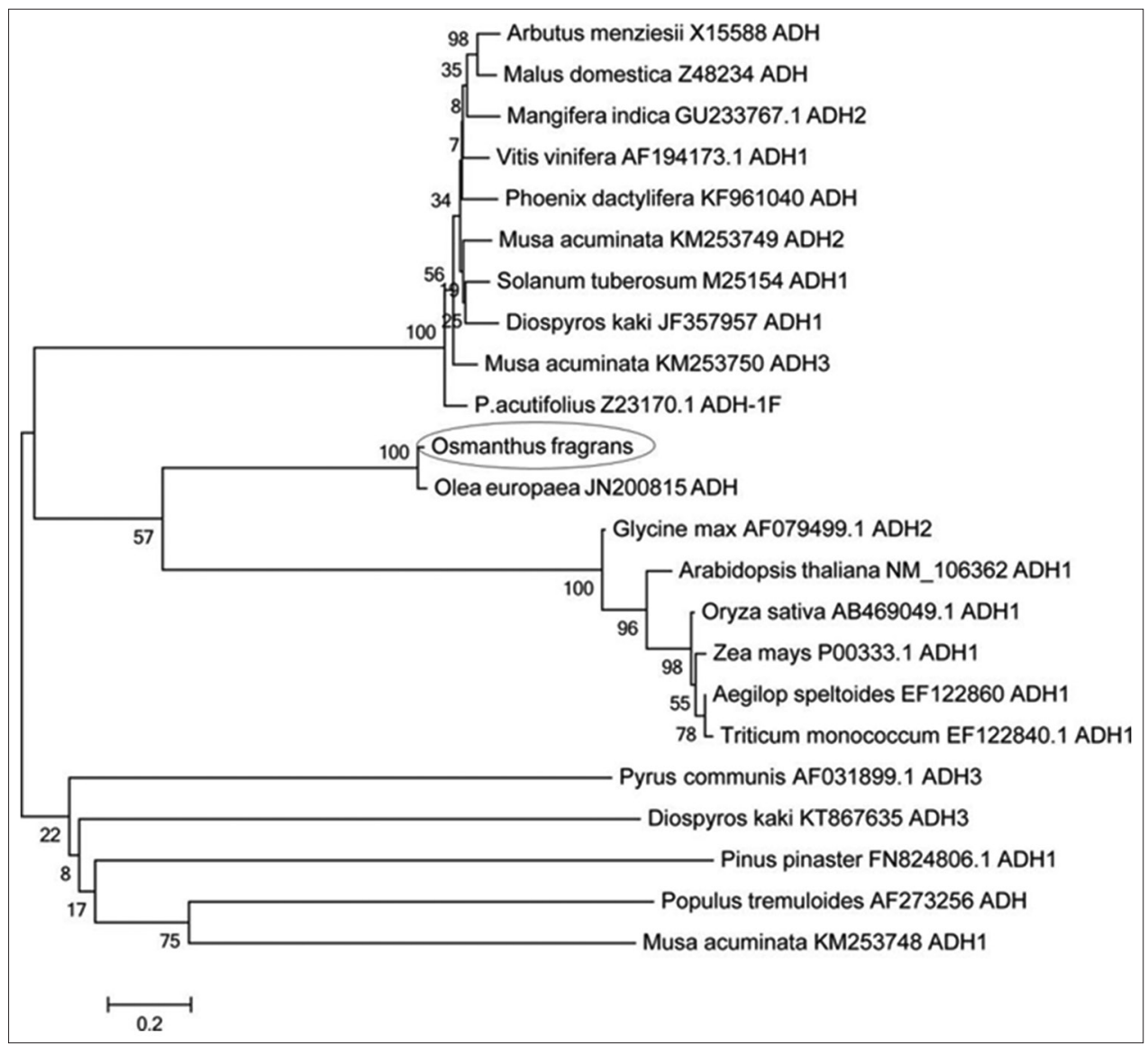

Fig 8. Phylogeny tree of ADHs from different plants.

Li et al., 2011). To explore the expression pattern of gene in the aroma synthesis pathway will be an effective method, because the difference of floral composition is significant among plants and the fragrance biosynthetic pathway is unclear. In this study, the full-length cDNA of $O f A D H$ have been cloned and sequence analyzed. The $O f A D H$ gene expression pattern, a trend of increasing from budding stage to blooming stage and then decreasing, was positive correlation with the aroma formation during flowering development ( $\mathrm{Li}$ et al., 2008). This conclusion was similar with observations that $\mathrm{ADH}$ gene had a certain promotion effect on aroma formation (Bellincontro et al., 2005). 


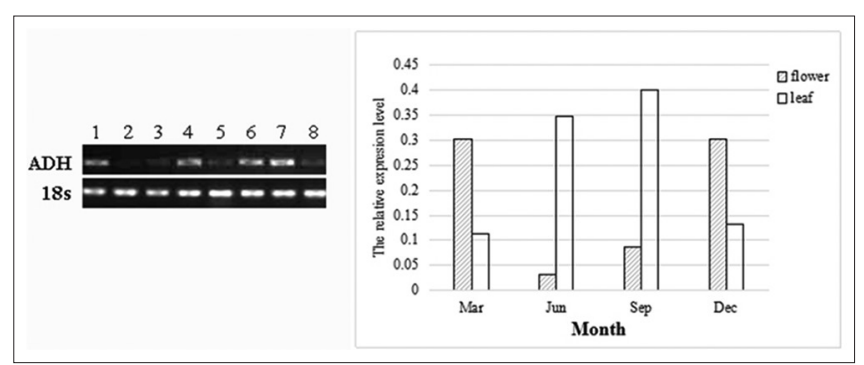

Fig 9. Expression level of $O$. fragrans var. semperflorens ADH gene in different months and tissues. 1-4: The OfADH expression of flowers in Mar, Jun, Sep, and Dec; 5-6=The OfADH expression of leaves in Mar, Jun, Sep, and Dec.

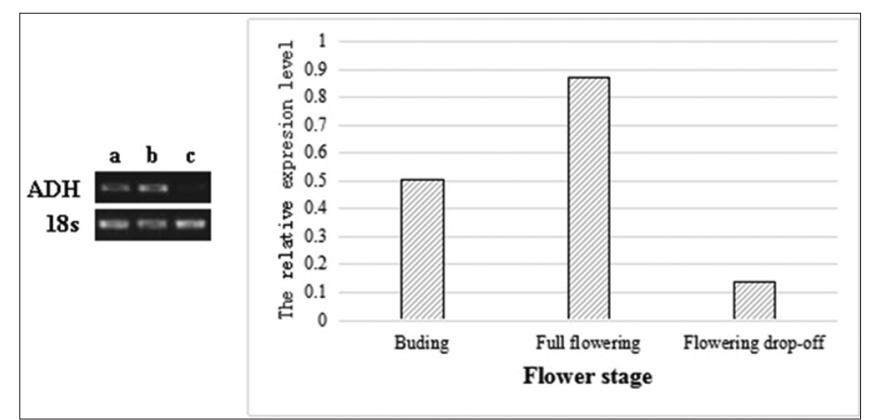

Fig 10. Expression level of ADH gene in different $O$. Fragrans flowering stages a: Budding stage; b: Full flowering; c: Flowering drop-off.

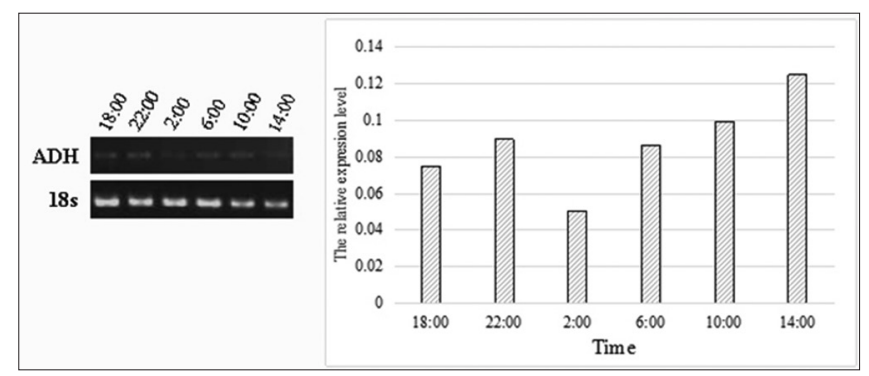

Fig 11. Expression level of OfADH gene in different times.

At the same time, a large of researches indicated that stress can promote expression of $\mathrm{ADH}$ in order to increase plant resistance and adaptability to the environment. ABA could induce ADH expression in Arabidopsis (De Bruxelles et al., 1996). The expression of ADH would increase in the water logging peanut roots (Liu et al., 2007). The certain concentration of salt stress could make ADH increasing significantly in rice roots (Liang, et al., 2012). ADH was up-regulated in the leaves of Banana Seedlings under cold stress (Jia et al., 2014). In this study, the expression level of $O f A D H$ was higher in June and September with relatively high temperatures than it was in March and December. When leaves of $O$. Fragrance were under high temperature stress, ADH gene expression would be increased because of the resistance response mechanism activation in order to avoid from heat damage. Many researches about functional components in the leaves, flowers (Ge et al., 2018a and 2018b), barks (Ge et al., 2017a and 2017b), stems (Ge et al., 2017c and 2017d), roots (Ge et al., 2017e; Chen et al 2017), fruits (Jiang et al., 2018a and 2018b), timbers (Peng et al., 2017a and 2017b) and byproducts (Liu et al., 2017; Xie et al., 2018) of plants showed that they contain many bioactive components (Peng et al., 2016; He et al., 2017; Cheng et al., 2017; Yang et al., 2017; Qin et al., 2017; Xu et al., 2018; Wang et al., 2018). This result was consistent with the resistance response function of ADH (Strommer et al., 2011).

This finding indicated that the $O f A D H$ has a certain function in the aroma synthesis pathway in flowers and heat shock response in leaves.

There is obvious functional diversity of $O f A D H$ in $O$. fragrance var. Semperflorens. The molecular mechanism of OfADH remains to be further research in order to find out how OfADH perform different function in flowers and leaves.

\section{ACKNOWLEDGEMENTS}

This work was supported financially by the Natural Science Fund of Hunan Province of China (2018JJ3874), the Key project of science and technology plan of Hunan province (2014FJ2004), the Central South University of Forestry and Technology talent introduction project (0119-104), and the talent project of Henan Agriculture University (Dangquan Zhang).

\section{REFERENCES}

Alpat, Ş., and A. Telefoncu. 2010. Development of an alcohol dehydrogenase biosensor for ethanol determination with toluidine blue o covalently attached to a cellulose acetate modified electrode. Sensors -Basel. 10(1): 748-764.

Bellincontro, A., F. Morganti, D. De Santis, R. Botondi, and F. Mencarelli. 2005. Inhibition of ethylene via different ways affects LOX and ADH activities and related volatile compounds in peach (cv. 'royal gem'). Acta. Hortic. 682: 445-452.

Bomatia, E. K., and J. P. Noela. 2005. Structural and kinetic basis for substrate selectivity in Populus tremuloides sinapy alcohol dehydrogenase, . Plant Cell. 17(5): 1598-1611.

Chao, N., S. X. Liu, B. M. Liu, N. Li, X. N. Jiang, and Y. Gai., 2014. Molecular cloning and functional analysis of nine cinnamyl alcohol dehydrogenase family members in Populus tomentosa, . Planta. 240(5): 1097-1112.

Charlton, M. 2004. Nonalcoholic fatty liver disease: A review of current understanding and future impact. Clin. Gastroenterol. Hepatol. 2(12): 1048-1058.

Chen, J. T., C. Y. Ni, J. W. Lou, and W. X. Peng. 2017. Molecules and functions of rosewood: Diospyros celebica. Arab. J. Chem. 11(6): 67-75..

Cheng, X., T. Yang, Y. Wang, B. Zhou, L. Yan, L. Teng, F. Wang, L. Chen, Y. He, K. Guo, and D. Zhang. 2017. New method for effective identification of adulterated Camellia oil basing on 
Camellia oleifera-specific DNA. Arab. J. Chem.11(6): 815-826.

Cheung, Y. H., O. Y. Lam, S. C. Lun, C. H. Pang, K. W. Wu, and W. K. Leung. 2017. Electrical stimulation for eggs and semen on Tavhypleus tridentatus collected from local restaurants in Hong Kong. Malays. J. Sustain. Agric. 1(2): 1.

Chung, H. J., and J. F. Robert. 1999. Arabidopsis alcohol dehydrogenase expression in both shoots and roots is conditioned by root growth environment. Plant Physiol. 121(2): 429-436.

De Bruxelles, C. L., W. J. Peacock, E. S. Dennis, and R. Dolferus. 1996. Abscisic acid induces the alcohol dehydrogenase gene in Arabidopsis. Plant Physiol. 11(1): 381-391.

Di Meglio, L. M., G. Staudt, H. Yu, and T. M. Davis. 2014. A phylogenetic analysis of the genus Fragaria (strawberry) using intron-containing sequence from the ADH-1 gene. PLoS One. 9(7): e102237.

Dudareva, N., and E. Pichersky. 2008. Metabolic engineering of plant volatiles. Curr. Opin. Biotechnol. 19(2): 181-189.

Fukuda, T., J. Yokoyama, T. Nakamura, I.J. Song, T. Ito, T. Ochiai, A. Kanno, T. Kameya, and M. Maki. 2005. Molecular phylogeny and evolution of alcohol dehydrogenase (Adh) genes in legumes. BMC Plant Biol. 5: 6-12.

Ge, S., L. Wang, Z. Liu, S. Jiang, X. Yang, W. Yang, W. Peng, and T. Cai. 2018a. Properties of nonvolatile and antibacterial bioboard produced from bamboo macromolecules by hot pressing. Saudi J. Biol. Sci. 25I: 474-478.

Ge, S., L. Wang, J. Ma, S. Jiang, and W. Peng. 2018b. Biological analysis on extractives of bayberry fresh flesh by gc-ms. Saudi J. Biol. Sci. 25I: 816-818.

Ge, S. B., X. M. Chen, D. L. Li, Z. L. Liu, H. Ouyang, W. X. Peng, and Z. F. Zhang. 2017a. Hemicellulose structural changes during steam pretreatment and biogradation of Lentinus edodes. Arab. J. Chem. 11(6): 771-781.

Ge, S. B., H. P. Gu, J. J. Ma, H. Q. Yang, S. C. Jiang, Z. L. Liu, and W. X. Peng. 2017b. Potential use of different kinds of carbon in production of decayed wood plastic composite. Arab. J. Chem. 11(6): 838-843.

Ge, S. B., Z. L. Liu, Y. Z. Furuta, and W. X. Peng. 2017c. Adsorption characteristics of sulfur solution by acticarbon against drinkingwater toxicosis. Saudi J. Biol. Sci. 24I: 1355-1360.

Ge, S. B., Z. L. Liu, Y. Z. Furuta, and W. X. Peng. 2017d. Characteristics of activated carbon remove sulfur particles against smog. Saudi J. Biol. Sci. 24I: 1370-1374.

Ge, S. B., Z. L. Liu, R. D. Li, Y. Furuta, and W. X. Peng. 2017 e. Desulphurization characteristics of bamboo charcoal from sulfur solution. Saudi J. Biol. Sci. 24I: 127-131.

Gottlieb, L. D. 1982. Conservation and duplication of isozymes in plants. Science. 216: 373-380.

He, H., J. Qin, X. Cheng, K. Xu, L. Teng, and D. Zhang. 2017. Effects of exogenous 6-BA and NAA on growth and contents of medicinal ingredient of Phellodendron chinense seedlings. Saudi J. Biol. Sci. 25(6): 1189-1195.

Imahon, Y., I. Kishioka, and K. Uemura. 2002. Physiological responses and quality attributes of Japanese pear 'kosui' fruit kept in low oxygen atmospheres. J. Hortic. Sci. Biotech. 77(6): 677-682.

Iyit, N. 2018. Modelling world energy security data from multinomial distribution by generalized linear model under different cumulative link functions. Open Chem. 16(1): 377-385.

Jacquelyn, L. M., S. Dvora, A. Efrat, W. Rachel, F. Amos, W. Abraham, S. Baruch, J. E. Howard, G. Joel, and H. Deborah. 2013. Alcohol metabolizing genes and alcohol phenotypes in an Israeli household sample. Alcohol Clin. Exp. Res. 37(11): 1872-1881.

Jia, C. H., Z. Q. Jin, S. H. Wang, Z. Wang, H. X. Miao, J. H. Liu, J. B. Zhang, and B. Y. Xu. 2014. Cloning and expression analysis of Maadh in banana under environmental stress. Agr. Sci. China. 30(7): 109-115.

Jiang, M., H. Wu, Z. Li, D. Ji, W. Li, Y. Liu, D. Yuan, B. Wang, and Z. Zhang. 2018. Highly selective photoelectrochemical conversion of carbon dioxide to formic acid. ACS Sustain. Chem. Eng. 6(1): 82-87.

Jiang, S. C., S. B. Ge, and W. X. Peng. 2017a. Molecules and functions of rosewood: Dalbergia Stevenson. Arab. J. Chem. 11(6): 782-792.

Jiang, S. C., S. B. Ge, X. Wu, Y. M. Yang, J. T. Chen, and W. X. Peng. 2017b. Treating n-butane by activated carbon and metal oxides. Toxicol. Environ. Chem. 99l: 753-759.

Khan, M. M. H. 2018. Occurrence, distribution, host preference and damage severity of red pumpkin beetle - a review. Malays. J. Halal Res. 1(1): 3-9.

Khan, S. A., S. M. Imam, A. Ahmad, S. H. Basha, and A. Husain. 2018. Synthesis, molecular docking with cox 1 \& and ii enzyme, admet screening and in vivo anti-inflammatory activity of oxadiazole, thiadiazole and triazole analogs of felbinac. J. Saudi Chem. Soc. 22(4): 469-484.

Kibria, A. A., Kamrunnessa, and M. M. Rahman. 2018. Extraction and evaluation of phytochemicals from green coconut (Cocos nucifera) shell. Malays. J. Halal Res. 1(2): 19-22.

Kluver, N., J. Ortmann, H. Paschke, P. Renner, and A. P. Ritter. 2014 Transient overexpression of adh8a increases allyl alcohol toxicity in zebrafish embryos. Plos One. 9(3): e90619.

Kmita, A., C. Fischer, K. Hodor, M. Holtzer, and A. Roczniak. 2018. Thermal decomposition of foundry resins: A determination of organic products by thermogravimetry-gas chromatographymass spectrometry (tg-gc-ms). Arab. J. Chem. 11(3): 380-387.

Li, X. Y., H. H. Tan, J. G. Fang, J. Han, and C. N. Song. 2011. Flavor compounds in fruits and research on them, Zhiwu Shengli Xuebao. Plant Physiol. 47(10): 943-950.

Li, Z. G., H. Cao, G. H. Zhu, J. R. Gao, and D. L. Shen. 2008. Study on chemical constituents of fragrance released from fresh flowers of three different Osmanthus fragrans lour. during different florescences, Linchan Huaxue Yu Gongye. Chemistry Chem. and Industry of. Forest For. Products. 28(3): 75-80.

Liang, Y., J. P. Yan, and X. L. Tan. 2012. Effects of salt-stress on expression of $\mathrm{ADH} 3$ and ALDH in radicle of rice (Oryze sativa), Hubei Nongye Kexue. Hubei J. Agric. Sci. 51(13): 2870-2872.

Liu, D. W., and L. Li. 2007. The response of alcohol dehydroganase activity \& and development of peanut roots to waterlogging \& and their relationship, Huasheng Xuebao. Peanut Sci. 36(4): 12-17.

Liu, H., and Z. Liu. 2010. Recycling utilization patterns of coal mining waste in China. Resour. Conserv. Recycl. 54(12): 1331-1340.

Liu, L., X. X. Cheng, W. W. Zhao, Y. H. Wang, X. Dong, L. L. Chen, D. Q. Zhang, and W. X. Peng. 2017. Systematic characterization of volatile organic components and pyrolyzates from Camellia oleifera seed cake for developing high value-added products. Arab. J. Chem.11(6): 802-814.

Min, T., X. R. Yin, Y. N. Shi, R. L. Zheng, Y. C. Yao, D. Grierson, B. F. Ian, and K. S. Chen. 2012. Ethylene-responsive transcription factors interact with promoters of ADH and PDC involved in persimmon (Diospyros kaki) fruit deastringency. J. Exp. Bot. 63(18): 6393-6405.

Naser, M., and R. Lawrey., 2018. A comparative analysis of the efficiency and productivity of selected food processing industries 
in Malaysia. Malays. J. Sustain. Agric. 2(1): 19-28.

Niculescu, M., T. Erichsen, and V. Sukharev. 2002. Quinohemo protein alcohol dehydrogenase-based reagentless amperometric biosensor for ethanol monitoring during wine fermentation. Anal. Chim. Acta. 463(1): 39-51.

Ning, W. B., J. H. Liu, C. H. Jia, B. Y. Xu, and Z. Q. Jin. 2009. Study on the relationship between activity of alcohol dehydrogenase and ethylene metabolism for postharvest banana fruit, Guoshu Xuebao. J. Fruit Sci. 26(3): 386-389.

Pan, H., R. Zhou, G. V. Louie, J. K. Mühlemann, E. K. Bomati, M. E. Bowman, N. Dudareva, R. A. Dixon, J. P. Noel, and X. Wang. 2014. Structural studies of cinnamoyl-coa reductase and cinnamyl-alcohol dehydrogenase, key enzymes of monolignol biosynthesis. Plant Cell. 26(9): 3709-3727.

Peng, W. X., S. B. Ge, Z. L. Liu, and Y. Furuta. 2017a. Adsorption characteristics of sulfur powder by bamboo charcoal to restrain sulfur allergies. Saudi J. Biol. Sci. 241: 103-107.

Peng, W. X., D. L. Li, M. L. Zhang, S. B. Ge, B. Mo, S. S. Li, and M. Ohkoshi. 2017b. Characteristics of antibacterial molecular activities in poplar wood extractives. Saudi J. Biol. Sci. 24I: 399-404.

Peng, W. X., Z. Lin, L. S. Wang, J. B. Chang, F. L. Gu, and X. W. Zhu. 2016. Molecular characteristics of Illicium verum extractives to activate acquired immune response. Saudi J. Biol. Sci. 23I: 348-352.

Qin, J., Y. Wang, G. He, L. Chen, H. He, X. Cheng, K. Xu, and D. Zhang. 2017. High-efficiency micropropagation of dormant buds in spine base of red pitaya (Hylocereus polyrhizus) for industrial breeding. Int. J. Agric. Biol. 19(1): 193-198.

Shen, Y., W. Mi, and Z. Zhang. 2017. A positioning lockholes of container corner castings method based on image recognition. Pol. Marit. Res. 24(SI): 95-101.

Speirs, J., E. Lee, K. Holt, Y. D. Kim, N. S. Scott, B. Loveys, and W. Schuch. 1998. Genetic manipulationZ of alcohol deshydrogenase levels in ripening tomato fruit affects the balance of some flavour aklehydes and alcohols. Plant Physiol. 117: 1047-1058.
Strommer, J. 2011. The plant ADH gene family. Plant J. 66(1): 128-142.

Sultana, T., S. Rahman, N. Naher, R. M. D. Masum, A. H. A. Ahmed, and R. Islam. 2018. Performance of fruit vegetables in summer under mahagony based agroforestry systems. Malays. J. Halal Res. 1(2): 08-14.

Susanne, B., K. Masaya, K. Miwako, K. Yoshiko, F. Akira, F. Peter, and W. Naoharu. 2010. Functional characterization of a carotenoid cleavage dioxygenase 1 and its relation to the carotenoid accumulation and volatile emission during the floral development of Osmanthus fragrans lour. J. Exp. Bot. 61(11): 2967-2977.

Vanessa, J. M., A. R. Vijay, K. Nnenna, K. John, M. S. Denise, L. F. Clifford, and E. T. Robert. 2014. Evaluation of the influence of alcohol dehydrogenase polymorphisms on alcohol elimination rates in African Americans. Alcohol Clin. Exp. Res. 38(1): 51-59.

Wang, L., S. Ge, Z. Liu, Y. Zhou, X. Yang, W. Yang, D. Li, and W. Peng. 2018. Properties of antibacterial bioboard from bamboo macromolecule by hot press. Saudi J. Biol. Sci. 25(3): 465-468.

Xie, Y., S. Ge, S. Jiang, Z. Liu, L. Chen, L. Wang, J. Chen, L. Qin, and W. Peng. 2018. Study on biomolecules in extractives of Camellia oleifera fruit shell by gc-ms. Saudi J. Biol. Sci. 25I: 234-236.

Xu, K., G. He, J. Qin, X. Cheng, H. He, D. Zhang, and W. Peng. 2018. High-efficient extraction of principal medicinal components from fresh Phellodendron bark (cortex phellodendri). Saudi J. Biol. Sci. 25(4): 811-815.

Yang, D., H. Zhang, K. Peng, L. Chen, H. He, X. Huang, J. Qin, and D. Zhang. 2016. Differential gene regulation of lipid synthesis in the developing seeds of two biodiesel tree species, Jatropha and Vernicia. Int. J. Agric. Biol. 18(6): 1143 -1152.

Zhang, Q., Y. Y. Tian, Y. E. Meng, Y. M. Li, H. J. Wang, L. Wang and X. S. Zhao. 2009. Research advance in genetic engineering of floral fragrance, Jiyin Zuxue yu Yingyong Shengwuxue. Genomics Appl. Biol. 28(1): 159-166.

Zheng, X., C. Hu, D. Spooner, J. Liu, J. Cao, and Y. Teng. 2011. Molecular evolution of Adh and leafy and the phylogenetic utility of their introns in pyrus (Rosaceae). BMC Evol. Biol. 11: 255. 